\title{
Perioperative Visual Deficit in a Patient Undergoing Dorsolumbar Spine Surgery
}

\author{
Ved Prakash Pandey ${ }^{1} \quad$ Arnab Dasgupta ${ }^{1} \quad$ Anurag Aggarwal ${ }^{1} \quad$ Sachin Jain ${ }^{1}$ \\ ${ }^{1}$ Department of Neuroanaesthesiology, Fortis Hospital, Noida, \\ Uttar Pradesh, India

\begin{abstract}
Address for correspondence Arnab Dasgupta, MBBS, MD, Department of Neuroanaesthesiology, Fortis Hospital, B-22, Sector 62, Noida 201301, Uttar Pradesh, India (e-mail: arnab.dasgupta@fortishealthcare.com).
\end{abstract}

\begin{abstract}
Perioperative visual loss (POVL) is a rare but potentially serious complication of longduration surgeries in prone position under general anesthesia. The mechanism of visual loss after surgery, and its incidence, is difficult to determine. It is primarily associated with cardiothoracic and spine surgeries. The proposed causes include corneal injury, retinal ischemia (central retinal artery occlusion/branch retinal artery occlusion [CRAO/ BRAO]), ischemic optic neuropathy (ION), and cortical blindness. A large, recent multicenter case-control study has identified risk factors associated with ION for patients undergoing spinal instrumentation surgery in prone position. These include male sex, obesity, use of Wilson's frame, long duration of anesthesia/surgery, larger estimated blood loss, and larger relative use of crystalloids for compensation of blood loss. This report describes a relatively healthy, 71-year-old female patient who developed significant visual impairment after thoracolumbar spine surgery in prone position under general anesthesia. The case raises dilemmas regarding the preoperative identification of patients who should be informed of the risk of POVL, and by whom.
\end{abstract}

\section{Introduction}

Perioperative vision loss (POVL) after nonocular surgery is a worrisome complication as it has significant impact on the patient's quality of life. The reported incidence of POVL varies from 0.05 to $1.3 \% .^{1}$ Cardiac surgeries (incidence $0.09 \%$ ) and spine surgeries (incidence as high as $0.2 \%)^{2}$ pose the highest risk of POVL. Other nonocular surgeries associated with POVL reports include orthopedic joint surgeries, ${ }^{3}$ endoscopic sinus/ nasal surgeries, ${ }^{4}$ obstetrics/gynecologic, and urologic surgeries. ${ }^{5}$ Etiologies of POVL ${ }^{6}$ include ischemic optic neuropathy (ION) that can be anterior (AION) or posterior (PION), and retinal ischemia, which may be due to central retinal artery occlusion (CRAO) or branch retinal artery occlusion (BRAO), cortical blindness (CB), and corneal abrasion (CA). In 2012, the American Society of Anesthesiologists' (ASA) Task Force on Perioperative Visual Loss, in its advisory, defined POVL as permanent or total loss of vision during spine surgery under general anesthesia (GA). ${ }^{7}$
We describe a patient who developed permanent postoperative visual impairment after spine surgery in prone position. Postoperative visual impairment was unanticipated, as known risk factors during preanesthetic workup were absent. Surgical risk factors, such as prolonged duration and extent of blood loss, were not anticipated.

\section{Case Report}

A 71-year-old female patient, $160 \mathrm{~cm}$ in height, weighing $65 \mathrm{~kg}$, was posted for dorsolumbar spinal decompression and fixation for D12-L1 fracture/collapse with kyphosis due to suspected Pott's spine. She had no other comorbidities and had no ophthalmologic complaints prior to surgery. On preoperative evaluation, she was accepted for GA in ASA physical status II. Preoperative laboratory values were essentially normal, with hemoglobin ( $\mathrm{Hb}$ ) level being $11.20 \mathrm{~g} / \mathrm{dL}$. She was administered injections of propofol, atracurium, and nalbuphine at induction, in standard doses, prior to intubation.

\section{received}

February 28, 2018 accepted after revision June 11, 2018 published online

August 29, 2018
DOI https://doi.org/

10.1055/s-0038-1667211.

ISSN 2348-0548.
Copyright $\odot 2018$ Indian Society of Neuroanaesthesiology and Critical Care
License terms

() (1) $\odot \circledast$ 
Anesthesia was maintained with $\mathrm{O}_{2}$, air, and isoflurane. Routine ASA monitoring was done, with additional invasive blood pressure monitoring. She was positioned prone on Allen's frame, taking due precautions. Intraoperatively, her eyes were examined every 30 minutes via reflector mirror of Allen's frame. Total duration of anesthesia was 6.5 hours, and the patient remained prone for 6 hours. Estimated blood loss was 3,500 to $3,800 \mathrm{~mL}$, which was managed with adequate crystalloids, colloids (500 mL volulyte), blood (2 units packed red blood cells), and blood products (4 units fresh frozen plasma). Hypotension, with systolic blood pressure of 50 to $70 \mathrm{~mm} \mathrm{Hg}$, necessitated the use of noradrenaline infusion. On completion of surgery, the patient was shifted to the neurosurgical intensive care unit (ICU) on mechanical ventilatory support, on noradrenaline infusion. Postoperative Hb was $6.5 \mathrm{~g} / \mathrm{dL}$, for which 2 more units of packed red cells were transfused, following which noradrenaline infusion was tapered. The patient was extubated the following day, around 24 hours after completion of surgery. On the second postoperative day, she complained of diminished vision in her left eye, without any associated pain. The loss of vision was partial, but progressive. Ophthalmologic opinion was obtained. On confrontation, left temporal hemianopia was present, and on funduscopy, changes suggestive of disc edema were seen. The patient was given a trial of intravenous methylprednisolone, $125 \mathrm{mg}$ twice daily for 48 hours. Three months after discharge, she still has visual impairment.

\section{Discussion}

Vision loss or deficit after surgery is a rare complication, with poor prognosis. In AION, it typically occurs 24 to 48 hours after surgery. ${ }^{8}$ The pressure in the central retinal artery is around 50 to $60 \mathrm{~mm} \mathrm{Hg}$, whereas intraocular pressure (IOP) in upright position is between 10 and $15 \mathrm{~mm} \mathrm{Hg}$, providing a perfusion pressure of approximately $50 \mathrm{~mm} \mathrm{Hg.}{ }^{9}$ IOP doubles when an anesthetized patient is made prone from supine, and then continues to rise with time, reaching $40 \mathrm{~mm} \mathrm{Hg}$ after 320 minutes. ${ }^{9}$ At an IOP of $60 \mathrm{~mm} \mathrm{Hg}$, blood flow to the optic nerve at the disc ceases. Risk factors for ION after spinal fusion surgery are:

- Male sex

- Obesity

- Use of Wilson's frame

- Prolonged duration of surgery and anesthesia: $\geq 6.5$ hours $^{7}$

- Significant intraoperative blood loss: $\geq 40 \%$ of estimated blood volume ${ }^{7}$

- Excessive crystalloid use for blood loss replacement

This case, presumed to have developed AION, highlights that postoperative visual deficit can occur following spine surgery in prone position, even if the patient does not have comorbidities that increase the risk of POVL. In our case, the prolonged duration and extent of blood loss could not be anticipated preoperatively, and consent for possible POVL was not obtained. During retrospective analysis, it was obvious that adequate units of blood and blood products had not been arranged. If more packed red blood cells had been arranged and transfused intraoperatively, it is possible that the risk of this complication could have been reduced. POVL can occur due to multiple etiologies, and there are no proven effective treatment modalities. Therefore, disclosure of the information and risks regarding POVL to the patient and his/ her relatives preoperatively, and obtaining the consent for the same, seems appropriate. The dilemma that arises from this case is whether one should obtain informed consent for POVL in all spine surgeries in prone position, including cases presumed to be at low risk preoperatively. The second issue relates to whether the surgeon, the anesthesiologist, or both should obtain the consent.

Depending on the suspected etiology, the investigations advised can include computed tomography (CT) of the head, funduscopy, and fluorescein angiography. It is important to differentiate between AION and PION, as prognosis is better in AION. AION presents as painless loss of vision, with the lesion in or adjacent to the optic disc, whereas PION is seen in the posterior portion of the optic nerve. Disc edema is noted in AION but not in PION.

Other mechanisms of POVL include retinal ischemia, due to CRAO/BRAO, resulting from external compression of the eye during surgery, and thrombotic or ischemic events within occipital lobes during surgery, which can result in cortical blindness. The prognosis of these types of POVL is usually not good, due to absence of any specific therapy.

\section{Conclusion}

Perioperative visual deficit or loss can occur in patients presumed to be at low risk on routine preoperative evaluation. There are unresolved issues on whether to obtain consent for POVL in all cases undergoing spine surgery in prone position, even if anticipated blood loss is not expected to be high and duration of surgery is expected to be less than 6.5 hours. If POVL is mentioned in the consent, would it be reasonable for both the surgeon and anesthesiologist to obtain the same? Thorough preoperative evaluation and preparation, intraoperative positioning of patients with head neutral or above heart level, aggressive correction of anemia, maintenance of hemodynamics, management of intravenous fluids, and optimization of duration of surgery are the steps that can be taken to decrease the incidence of this dreaded complication.

\section{Conflict of Interest}

None.

\section{References}

1 Kitaba A, Martin DP, Gopalakrishnan S, Tobias JD. Perioperative visual loss after nonocular surgery. J Anesth 2013;27(6):919-926

2 Nickels TJ, Manlapaz MR, Farag E. Perioperative visual loss after spine surgery. World J Orthop 2014;5(2):100-106

3 Shen Y, Drum M, Roth S. The prevalence of perioperative visual loss in the United States: a 10-year study from 1996 to 2005 of spinal, orthopedic, cardiac, and general surgery. Anesth Analg 2009;109(5):1534-1545

4 Halvorsen H, Ramm-Pettersen J, Josefsen R, et al. Surgical complications after transsphenoidal microscopic and endoscopic 
surgery for pituitary adenoma: a consecutive series of 506 procedures. Acta Neurochir (Wien) 2014;156(3):441-449

5 Moslemi MK, Soleimani M, Faiz HR, Rahimzadeh P. Cortical blindness after complicated general anesthesia in urological surgery. Am J Case Rep 2013;14:376-379

6 De la Garza-Ramos R, Samdani AF, Sponseller PD, et al. Visual loss after corrective surgery for pediatric scoliosis: incidence and risk factors from a nationwide database. Spine J 2016;16(4):516-522

7 American Society of Anesthesiologists Task Force on Perioperative Visual Loss. Practice advisory for perioperative visual loss associated with spine surgery: an updated report by the American Society of Anesthesiologists Task Force on Perioperative Visual Loss. Anesthesiology 2012;116(2):274-285

8 Lee L, Roth S, Todd M, et al; Postoperative Visual Loss Study Group. Risk factors associated with ischemic optic neuropathy after spinal fusion surgery. Anesthesiology 2012;116(1):15-24

9 O'Driscoll A, White E. Care of the eye during anaesthesia and intensive care. Anaesth Inten Care Med 2017;18(1):47-51 